\title{
Dysphagia between Iatrogenic Neuroleptics and Organicity: About A Clinical Case
}

\author{
Hajar Rguibi*, Aya Chaara, Siham Belbachir, Abderrazzak Ouanass
}

University psychiatric Hospital Arrazi in Sale, Faculty of Medicine and Pharmacy, Mohammed V University, Rabat, Morocco

DOI: $10.36347 /$ sjmcr.2020.v08i07.011

| Received: 06.07.2020 | Accepted: 13.07.2020 | Published: 24.07.2020

*Corresponding author: Hajar Rguibi

Abstract

Dysphagia is a serious condition in which swallowing problems interfere with the patient's ability to eat, resulting pneumonia by aspiration, malnutrition, suffocation and asphyxia. It is a common symptom in the general population, and even more in the psychiatric patient. Antipsychotic drugs have traditionally been associated with extrapyramidal symptoms; these can be in the form of dysphagia, esophageal dysmotility or aspiration, which may not be recognized as an effect. The symptom of dysphagia may also appear in the psychiatric patient as in all other patients in a pathological context such as myasthenia gravis hence the difficulty to take into account dysphagia of iatrogenic origin and organic origin. We report the case of a patient with schizophrenia treated with neuroleptics first generation who presented with dysphagia with regurgitation, dysphonia and deterioration of the general state. The symptom was initially misdiagnosed as extrapyramidal side effect of neuroleptics but which did not give way even when molecules were stopped. Myasthenia gravis has been confirmed as the etiology of this dysphagia after an injury assessment.

Keywords: Dysphagia, Myasthenia, neuroleptics, Schizophrenia.

Copyright @ 2020: This is an open-access article distributed under the terms of the Creative Commons Attribution license which permits unrestricted use, distribution, and reproduction in any medium for non-commercial use (NonCommercial, or CC-BY-NC) provided the original author and source are credited.

\section{INTRODUCTION}

Dysphagia is defined as difficulty in swallowing or getting the food bolus from the oral cavity to the stomach. It indicates a difficulty in the initiation of swallowing, or a cervical blockage, sometimes associated with oro-nasal regurgitation, false routes, or the perception of an oral or pharyngeal residue after swallowing [1-3]. It can be secondary to an upper pharyngo-esophageal obstruction, to a neurological disorder, or more rarely to a myopathy of the striated muscles in the cervical esophagus.

It is a symptom which is observed in a multitude of medical problems which can cause false routes, at the origin of multiple complications up to death.

There are several etiologies of dysphagia whose diagnosis is difficult to make when they are not obvious. This sign may be inaugural alongside the other bulbar signs (dysphonia, chewing disorders) in $15 \%$ of cases of myasthenia gravis. In addition, this sign can precede a myasthenic crisis requiring resuscitation care or even be the cause of false routes, responsible of the morbidity and mortality from this disease.
Dysphagia is a symptom, common in the general population, and also known in psychiatric patients before the advent of psychotropic drugs [4]. It is now recognized that neuroleptics may cause and/or worsen dysphagia $[5,6]$. The myastenic cause of dysphagia is rarely mentioned in the psychiatric patient, especially patients suffering from schizophrenia. Indeed, the comorbidity of myasthenia- schizophrenia is rare. Gittleson and Richardson have suggested a possible mutual antagonism between the two diseases, since an increase in muscle tone is frequently observed in schizophrenia [35], but the mechanism of this comorbidity is not yet known.

\section{The objective of our work is to}

- Present a clinical case of dysphagia in a patient who was hospitalized in the B psychiatric unit of the Arrazi Hospital in Sale-Morocco.

- Discuss the etiology of myasthenia gravis in this patient under neuroleptic treatment.

- Demonstrate the diagnostic difficulties encountered and the relationship with neuroleptics.

- Evoke the difficulty of some pharmacological prescriptions in the mental patient suffering from myasthenia gravis. 


\section{Patient and Observation}

Mr AA, 56 years old, single, without profession, living in Rabat, followed in psychiatry at the Arrazi Hospital in Sale since 1992 at the age of 29 for schizophrenia according to DSM5 criteria, with history of three hospitalizations all under the request of a third part or on police requisition, for violence and aggression, due to the discontinuation of his antipsychotic treatment.

His last hospitalization dating back to July 2019, following a therapeutic discontinuation of 3 months. He attempted to kill his sister due to hallucinations ordering him. The patient also allegedly threatened his brother and neighbors with death. He was allegedly found by the police officers to be wandering and uninhibited. The psychiatric examination identified dissociative syndrome, delusional syndrome, hallucinatory syndrome with troubles of judgment and insight. During this hospitalization, psychotropic drugs were reintroduced when reaching high doses: Haloperidol $12 \mathrm{mg}$ / day, Chlorpromazine $300 \mathrm{mg} /$ day and diazepam $15 \mathrm{mg} /$ day.

During this stay, dysphagia with solids and liquids with false routes and regurgitation with deterioration of the general state is noted, aggravated by the increase in treatment after almost ten days of hospitalization. Diazepam was discontinued and an anticholinergic corrector was introduced but without any clinical improvement. The patient was initially referred urgently to a specialist in gastrology and whose abdominal examination and upper digestive endoscopy did not show any anatomical lesions. The patient was refered to an otorhinolynrogolist because of the association of dysphagia with a phonation disorder and a nasal voice.

An otorhinolynrogology examination and upper airway endoscopy were performed, revealing no abnormality. Thus, late laryngeal dyskinesia induced by neuroleptics has been suspected, hence the discontinuation of conventional neuroleptics (haloperidol and chlorpromazine) and the setting of the patient on atypical antipsychotic: olanzapine at a dose of $5 \mathrm{mg} /$ day, but dysphagia and dysphonia have persisted. The patient subsequently received advice from a neurologist. The specialized neurological examination showed tiredness, asthenia, a nasal voice, osteotendinous reflexes were weak in all four limbs. The ice cube test was done and found to be positive. The patient also benefited from an electromyogram which highlighted the presence of bilateral spinal diminutions. The myasthenic score improved after injection of prostigmine.

The patient was put on pyridostigmine bromide with improvement of his condition while maintaining a low dose of olanzapine $5 \mathrm{mg} /$ day. Other explorations such as the thoracic scanner for thymic hyperplasia, the assay of antibodies against acetylcholine receptors have been requested.

The patient's psychiatric state had decompensated with olanzapine with the reappearance of delusional and hallucinatory symptoms which necessitated the change to risperidone at an initial dose of $2 \mathrm{mg}$ / day while ensuring regular monitoring.

\section{DISCUSSION}

Myasthenia gravis (MG) is an autoimmune disease characterized by a block in the neuromuscular junction, caused by autoantibodies directed against acetylcholine receptors (RAch).

This disease is marked clinically by a fluctuating motor deficit, exacerbating on exertion and yielding at rest. The dysphagia did not return. The diagnosis retained is myasthenia gravis.

The evolution was manifested by interspersed episodes that can endanger the vital prognosis by affecting the respiratory muscles.

Dysphagia can be an initial sign in around 15\% of myasthenia gravis [7].

Nevertheless, dysphagia of myasthenic origin in the psychiatric troubles is rarely mentioned. It is mainly linked to an iatrogenic cause following the use of neuroleptics.

However, dysphagia secondary to antipsychotics can occur regardless of the class of the molecule. According to the literature, this symptom has been associated with many first generation antipsychotics, including chlorpromazine and haloperidol, in patients aged from 38 to 79 years $[8,9]$.

Etiological research is important to carry out.

This case describes a patient with schizophrenia who presented dysphagia and was initially wrongly diagnosed as an iatrogenic cause (extrapyramidal effect of neuroleptics). The diagnosis of myasthenia gravis with functional evidence was later confirmed. In retrospect, it appeared that his dysphagia worsened while increasing conventional neuroleptics before their discontinuation. The resolution of his dysphagia was not observed despite the discontinuation of conventional neuroleptics.

There are four main etiologies of dysphagia in psychiatric patients. Those are:

- Extrapyramidal syndrome [10, 11]. Dysphagia is present in 50\% of patients with Parkinson's disease, it is also very common in secondary parkinsonian syndromes without being able to give a prevalence [12]. It is most often associated with another symptom of extrapyramidal syndrome, but can also be isolated, making its diagnosis more difficult [13, 14]. This dysphagia is called bradykinesia, it 
indicates a slowing of the oral phase and the pharyngeal reflex [13].

- Tardive dyskinesia [10]: The oropharyngooesophageal form is the most frequent and can be isolated.

- Esophageal dyskinesias appear after three months of treatment with neuroleptics [14]; they correspond to random and asynchronous esophageal movements with the appearance of regurgitation, false routes, and inhalations.

- More rarely, acute laryngeal or esophageal dystonia $[15,16]$, associated or not with orofacial dyskinesias, is characterized by a reduction in oesophageal muscle contractions and esophageal hypertonia in the upper sphincter, it is rarely secondary to atypical antipsychotics with dependent dose.

- Polyphagia [10] or binge eating, which is common in psychotic patients, is also a great provider of false routes, without being dysphagia strictly speaking.

- Some conditions can be revealed by high dysphagia; should be systematically mentioned: myasthenia gravis, amyotrophic lateral sclerosis (ALS), and some rare myopathies [17-19].

- We must systematically look for the risk factors favoring dysphagia: poor dental condition, neurological pathologies, xerostomia, polypharmacy, advanced age, gastroesophageal reflux, sedative drugs, depression, etc [10].

Sometimes dysphagia is caused by the onset of hyper salivation that occurs when using clozapine or clonazepam [20-22]. This hypersalivation can sometimes be the cause of aspiration. More rarely, this phenomenon may be observed with the intake of olanzapine, haloperidol, lithium, donepezil or physostigimine [20].

Our case of dysphagia was secondary to myasthenia gravis. Myasthenia gravis is initiated by dysphagia in 6 to $17 \%$ of cases with a prevalence of 4/100.000 inhabitants [23]. The diagnosis is based on other signs of muscle fatigue, a thymoma and especially anti-acetylcholine antibodies present in almost $85 \%$ of cases.

Schizophrenia is associated with a prevalence of approximately $50 \%$ with autoimmune disorders during life [24]. However, myasthenia-schizophrenia comorbidity is rare according to studies in the literature and poses a challenge in the management of both [35]. So far, less than 10 cases of this rare association have been reported in the literature [25].

The facts show that the age of beginning of myasthenia gravis varies from 4 years to 69 years, two thirds of these patients are women.
The reason for the low comorbidity of myasthenia gravis and schizophrenia is unknown compared to the high comorbidity of myasthenia gravis with other psychiatric disorders. A study found that 38 $(51 \%)$ of 74 patients with myasthenia gravis were diagnosed with psychiatric disorders, including adjustment disorders with depressed and mixed mood with emotional characteristics (19\%), affective disorders $(13.5 \%)$ and personality disorder (18\%), but no case of schizophrenia has been identified [36].

Several drugs, including some antipsychotics, have been shown to worsen the symptoms of myasthenia gravis. Our patient, whose undiagnosed myasthenia gravis was exposed by antipsychotic drugs used to treat a psychotic relapse of schizophrenia and whose symptoms were first attributed to the extrapyramidal side effects associated with antipsychotics.

Previous studies have indicated that isolated bulbar symptoms occurred in less than $25 \%$ of myasthenia gravis with no typical presentation of ptosis or general weakness. Oropharyngeal muscle weakness produces dysphonia and dysphagia with weakness of the palatal muscles, and the nasal voice develops when air escapes through the nose [37], which can be misdiagnosed as tardive dyskinesia in patients with schizophrenia because they are exposed to antipsychotics.

Benzodiazepines, anticholinergics and certain antipsychotics can exacerbate dysphagia and can lead to a myasthenia gravis crisis, leading to respiratory failure.

It should be noted that most of those who had myasthenia gravis in schizophrenic patients were of the general type and only one case was associated with a thymoma. These cases were diagnosed due to the exacerbation of myasthenia gravis during treatment with antipsychotics. Our patient had no history of thymoma.

Many classes of drugs are well documented to cause clinical worsening of myasthenia gravis or unmasking an undiagnosed disease. These include several drugs commonly used in the treatment of psychiatric patients such as antipsychotics (Phenothiazines: Fluphenazine, Chlorpromazine ...), mood stabilizers (carbamazepine, lithium), benzodiazepine [26] and antidepressants [27].

Current statistics indicate that the first and second generation of antipsychotics have the potential to worsen symptoms in patients with myasthenia gravis with a higher incidence in those with maximum anticholinergic properties. Although the anticholinergic effect of antipsychotic drugs is mainly at the muscarinic receptor level, some also have limited but significant activity at the nicotinic receptor level [28]. 
The most common antipsychotics involved are: chlorpromazine, pimozide, thioridazine, clozapine, olanzapine, haloperidol [29] and recently quetiapine in a case report [30].

Four mechanisms have been described to explain the interaction of these drugs with myasthenia gravis:

- Inhibition of neuronal transmission at the presynaptic level;

- Lack of acetylcholine release (possibly linked to the inhibition of calcium influx in the presynaptic terminations)

- Increased production of autoantibodies at the nicotinic postsynaptic acetylcholine receptors.

- Prevention of the transmission of potential action beyond the postsynaptic terminations secondary to changes in the permeability to postsynaptic ions $[31,32]$.

Chlorpromazine was the first to produce increased muscle weakness in a schizophrenic patient with myasthenia gravis by McQuillen in 1963 [5]. The drug has been shown to combine the presynaptic and postsynaptic neuromuscular blocking effect in some studies; by inhibiting the release of acetylcholine and demonstrating competitive inhibition of acetylcholine receptors similar to the blocking of d-tubocurarine; sometimes called a membrane stabilizer effect $[12,14]$. Risperidone is a second generation antipsychotic (SGA) which acts as an antagonism of the D2 and 5-HT2A receptors and used to control delusions, hallucinations, agitation and aggressiveness in various psychotic disorders. This antipsychotic has not been linked to the worsening of myasthenia gravis in the literature. In a study by Chew et al; risperidone has shown fewer anticholinergic side effects at therapeutic doses compared to other antipsychotics like clozapine or olanzapine.

In our case, the patient received risperidone after improvements in the signs of myasthenia gravis to stabilize the patient psychiatrically.

In the literature, the antipsychotic drugs which are safer in myasthenia gravis are mainly drugs predominantly dopaminergic, for example: paliperidone and amisulpride [33].

The principles of the therapeutic management of myasthenia gravis are essentially based on optimizing the functioning of the neuromuscular junction by reducing the level of pathogenic antibodies and improving neuromuscular transmission. The general aim of treatment is ideally to achieve a return to normal life as quickly as possible, at the cost of the minimum of side effects and if possible at the lowest cost.
Symptomatic

treatments

and

immunoregulatory treatments acting in the short or long term constitute the therapeutic arsenal of myasthenia gravis.

Complications of dysphagia regardless of the cause are represented by: inhalation, pneumonia, reactive anorexia, weight loss and the most serious is death by suffocation [33].

The symptom of dysphagia is often underestimated by medical doctors in a hospital environment because it is rarely declared spontaneously by patients.

The management of dysphagia due to myasthenia gravis in patients suffering from schizophrenia must take into account some antipsychotic drugs aggravating myasthenia gravis. It must also limit the combinations of anticholinergic drugs and dopamine blockers with an adequate riskbenefit balance assessment.

\section{CONCLUSION}

Dysphagia in patients with psychiatric illnesses such as schizophrenia is a problem that can sometimes be complex if present for a long time and can be transient or chronic depending on the etiology involved.

Several cases of dysphagia in patients with psychiatric illness have been linked to the extrapyramidal effects of antipsychotics of first and second generation, while missing an organic cause such as myasthenia gravis. The latter is an essentially clinical diagnosis. Diagnostic and therapeutic difficulties can be observed in the mentally ill, hence the need to recommend preventive measures as a first resort. Particular attention must be paid by the caregiver in order to provide prompt treatment and regular monitoring while taking into consideration the serious risk of deterioration of dysphagia when it is secondary to myasthenia gravis as well as the risk of psychopathological decompensation caused by a modification of the pharmacological treatment.

Caution and patience are often the key to the success of such treatment modifications [34].

\section{Competing Interests: No conflict of Interest}

\section{REFERENCES}

1. Marta M, Laia R. Systematic review of case reports of oropharyngeal dysphagia following the use of antipsychotics. Gastroenterología y Hepatología, 2019 April; 42(4):209-227.

2. McCarthy RH, Terkelsen KG. Dysfonction œsophagienne chez deux patients après traitement à 
la clozapine. J Clin Psychopharmacol. 1994; 14(4):281.

3. Bourgeois M. Les dyskinésies tardives des neuroleptiques. Psychol Med. 1987; 19(10):1731-4.

4. Lin TW, Lee BS, Liao YC, Chiu NY, Hsu WY. Dosage élevé de dysphagie induite par l'aripiprazole. Int J Eat Disord. 2011; 45(2):305-6.

5. Casadebaig F, Philippe A. Mortalité chez des patients schizophrènes. Trois ans de suivi d'une cohorte. Encéphale. 1999; 25:329-37.

6. Fioritti A, Giaccotto L, Melega V. Choking incidents among psychiatric patients: retrospective analysis of thirty-one cases from the West Bologna psychiatric wards. Can J Psychiatry. 1997; 42:515-20.

7. Tanaka L, Gharzouli I, Cloutier T, Salvan D. Dysphagie aiguë inaugurale d'une crise myasthénique nécessitant une prise en charge réanimatoire. Annales françaises d'Oto-rhinolaryngologie et de Pathologie Cervico-faciale. 2014 October; 131(4):49.

8. Fioritti A, Giaccotto L, Melega V. Incidents d'étouffement chez les patients psychiatriques: analyse rétrospective de trente et un cas dans les services psychiatriques de l'ouest de Bologne. Can J Psychiatry. 1997; 42(5):515-50.

9. Dziewas R, Warnecke T, Schnabel M, Ritter M, Nabavi DG, Schilling M. Dysphagie induite par les neuroleptiques : rapport de cas et revue de la littérature. Dysphagie. 2007; 22(1):63-7.

10. Fioritti A, Giaccotto L, Melega V. Choking incidents among psychiatric patients: retrospective analysis of thirty-one cases from the West Bologna psychiatric wards. Can J Psychiatry. 1997; 42:515-20.

11. Sokoloff LG, Pavlakovic R. Neuroleptic-induced dysphagia. Dysphagia. 1997; 12:177-9.

12. Edwards LL, Pfeiffer RF, Quigley EM, Hofman R, Balluff M. Gastrointestinal symptoms in Parkinson's disease. Movement disorders: official journal of the Movement Disorder Society. 1991;6(2):151-6.

13. Dziewas R, Warnecke T, Schnabel M. Neurolepticinduced dysphagia: case report and literature review. Dysphagia, 2007; 22:63-7.

14. Stewart JT. Reversible dysphagia associated with neuroleptic treatment. J Am Geriatr Soc. 2001; 49:1260-1.

15. Mann SC, Cohen MM, Boger WP. The danger of laryngeal dystonia. Am J Psychiatry. 1979; 136:1344-5

16. Mann SC, Cohen MM, Boger WP. The danger of laryngeal dystonia. Am J Psychiatry. 1979; 136:1344-5.

17. Moss HB, Green A. Neuroleptic-associated dysphagia confirmed by esophageal manometry. Am J Psychiatry. 1982; 139:4.

18. Achem SR, DeVault KR, Dysphagia in aging, J Clin Gastroenterol. 2005; 39:357-71.
19. Cook IJ, Kharilhas PJ. AGA technical review on management of oropharyngeal dysphagia Gastroenterology. 1999; 116: 455-78.

20. Pouderoux PH. Troubles de la déglutition : étiologies et prise en charge. Hepato-Gastro 1999; 6:247-57.

21. Szabadi E, Tavernor S. Hypo- and hypersalivation induced by psychoactive drugs. CNS Drugs. 1999; 11(6):449-66.

22. Darydov L, Botts SR. Clozapine-induced hypersalivation. Ann Pharmacother. 2000; 34:662-5.

23. Szabadi E, Tavernor S. Hypo- and hypersalivation induced by psychoactive drugs. CNS Drugs. 1999; 11(6):449-66.

24. Schon F, Drayson M, Thompson RA. Myasthenia gravis and elderly people Age Ageing. 1996; 25:5658 .

25. Cunning S. When the Dx is myasthenia gravis. RN. 2000; 63:26-30.

26. She S, Yi W, Zhang B, Zheng Y. Worsening of Myasthenia Gravis After Administration of Antipsychotics for Treatment of Schizophrenia: A Case Report and Review of Literature. J Clin Psychopharmacol. 2017.

27. Eaton WW, Byrne M, Ewald H, et al. Association of schizophrenia and autoimmune diseases: linkage of Danish national registers. Am J Psychiatry. 2006; 163:521-528.

28. Silvestri N, Wolfe GI. Myasthenia gravis. Semin Neurol. 2012; 32:215-26.

29. Nguyen Q, Yang J, Miledi R. Effects of atypical antipsychotics on vertebrate neuromuscular transmission. Neuropharmacology. 2002;42:670-676.

30. Wittbrodt ET. Drugs and myasthenia gravis. An update. Arch Intern Med. 1997; 157:399-408.

31. Vincent A, Palace J, Hilton-Jones D. Myasthenia gravis. Lancet. 2001; 357:2122-2128.

32. Karcic AA. Drugs that can worsen myasthenia gravis. Postgrad Med. 2000; 108(2):25.

33. She S, Yi W, Zhang B, Zheng Y. Worsening of Myasthenia Gravis After Administration of Antipsychotics for Treatment of Schizophrenia: A Case Report and Review of Literature. J Clin Psychopharmacol. 2017.

34. La dysphagie chez l'adulte ; choisir la bonne voie; Janvier 2008, L'inhalo, 24.

35. Gittleson NL, Richardson TD. Myasthenia gravis and schizophrenia - a rare combination. The British Journal of Psychiatry. 1973 Mar;122(568):343-4.

36. Magni M. Dizionario delle parole straniere nell'Italiano attuale. De Vecchi; 1988.

37. Sanders D, Massey J, Juel V. MuSK antibody positive myasthenia gravis: response to treatment in 31 patients. InNeurology 2007 Mar 20 (Vol. 68, No. 12, pp. A299-A299). 530 WALNUT ST, PHILADELPHIA, PA 19106-3621 USA: LIPPINCOTT WILLIAMS \& WILKINS. 